\title{
CBX3 is associated with metastasis and glutathione/ glycosphingolipid metabolism in colon adenocarcinoma
}

\author{
Xiaoling Zhong ${ }^{1 \#}$, Jun $\mathrm{Ni}^{2 *}$, Zhijun Jia ${ }^{3 \#}$, Hong Yan ${ }^{4}$, Ying Zhang ${ }^{5}$, Yunyun Liu ${ }^{6,7}$ \\ ${ }^{1}$ Department of Blood Transfusion, Nanjing Drum Tower Hospital, The Affiliated Hospital of Nanjing University Medical School, Nanjing, China; \\ ${ }^{2}$ Department of Clinical Laboratory, Nanjing Drum Tower Hospital, The Affiliated Hospital of Nanjing University Medical School, Nanjing, China; \\ ${ }^{3}$ Department of Nuclear Medicine, Nanjing Drum Tower Hospital, The Affiliated Hospital of Nanjing University Medical School, Nanjing, China; \\ ${ }^{4}$ Department of Clinical Laboratory, The Second Affiliated Hospital of Nanjing Medical University, Nanjing, China; ${ }^{5}$ Department of Obstetrics \\ and Gynecology, Nanjing Drum Tower Hospital, The Affiliated Hospital of Nanjing University Medical School, Nanjing, China; ${ }^{6}$ Department of \\ Pathology, The Fourth Affiliated Hospital of Nanjing Medical University, Nanjing, China; ${ }^{7}$ Department of Pathology, Nanjing Medical University, \\ Nanjing, China \\ Contributions: (I) Conception and design: H Yan; (II) Administrative support: Y Zhang; (III) Provision of study materials or patients: Y Liu; (IV) \\ Collection and assembly of data: X Zhong; (V) Data analysis and interpretation: Z Jia, J Ni; (VI) Manuscript writing: All authors; (VII) Final approval \\ of manuscript: All authors. \\ \#These authors contributed equally to this work. \\ Correspondence to: Hong Yan. Department of Clinical Laboratory, The Second Affiliated Hospital of Nanjing Medical University, Nanjing, China. \\ Email: yanhong@njmu.edu.cn; Ying Zhang. Department of Obstetrics and Gynecology, Nanjing Drum Tower Hospital, The Affiliated Hospital of \\ Nanjing University Medical School, Nanjing, China. Email: zhy7359@sina.com; Yunyun Liu. Department of Pathology, Nanjing Medical University, \\ Nanjing, China. Email: zjyunyun1314@sina.com.
}

Background: Metastasis is the major cause of colon adenocarcinoma (COAD) mortality. Increasing studies demonstrated that the epigenetics and downstream expression change of pivotal genes may act as a major role in promoting COAD progression and metastasis. Therefore, identifying the dysregulation of key genes associating with COAD metastasis may provide a new strategy for the discovery of potential treatment targets.

Methods: This study included a single-cell RNA sequencing profile consisting of 17,469 tumor cells derived from 23 samples, and 326 COADs available from The Cancer Genome Atlas (TCGA), etc. The study was performed using comparative analysis to characterize the role of $C B X 3$ in COAD metastasis and progression.

Results: This study revealed that the mRNA level of Chromebox homolog 3 (CBX3) in the metastatic COAD was significantly higher than that of the primary COAD and normal colon tissues (Wilcoxon's rank-sum test, $\mathrm{P}<0.05)$. Activation of $C B X 3$ was involved in regulating an interaction network consisting of $C C T 6 A, L S M 5$, and GGCT, etc., which may subsequently participate in glutathione metabolism. Besides, $C B X 3$ also exhibited a negative correlation with glycosphingolipid metabolism, which may associate with the regulation of $\mathrm{CBX} 3$ on DNA methylation. Clinical data analysis demonstrated that patients with high $C B X 3$ mRNA levels showed a nearly 2-fold shorter overall survival time than the control group (hazard ratio =1.59; likelihood ratio test, $\mathrm{P}=0.04)$.

Conclusions: Our study demonstrated that $C B X 3$ overexpression is associated with COAD metastasis. $C B X 3$ downstream regulation network involves in TCP1 complex, LSM family, and glutathione metabolism, which may provide a potential target for suppressing tumor metastasis.

Keywords: Colon adenocarcinoma (COAD); metastasis; CBX3; glutathione metabolism; prognosis

Submitted Dec 07, 2021. Accepted for publication Feb 18, 2022.

doi: 10.21037/jgo-22-97

View this article at: https://dx.doi.org/10.21037/jgo-22-97 


\section{Introduction}

Metastasis is a major cause of death for patients with colon adenocarcinoma (COAD) (1). Current standard treatments, including surgery and chemotherapy, have prolonged overall survival time of COAD patients, but more than $50 \%$ still experience metastasis after treatment (2). The identification of potential targets for inhibiting tumor metastasis and improving prognosis is urgently needed. Previous studies mainly focused on using whole genome/exome sequencing and found that COAD progression is an accumulation of mutations in pivotal genes regulating tumor-associated pathways. For instance, the APC mutation, which is found in more than $70 \%$ of colon cancer patients, is thought to be an initiating event for carcinogenesis in COAD (3). The TP53 and KRAS mutations may promote COAD progression to resistance to chemotherapy (4) and cetuximab treatment (5), respectively. However, recent research pointed out that the epigenetics and downstream change of gene expression, which endows tumors with plasticity and fitness to microenvironment stress (6), act as a major role in promoting COAD progression, metastasis, and recurrence. Increasingly, studies are concluding that tumor development is similar to embryonic development $(7,8)$. Key development signaling pathways, such as the Wnt pathway, have been found to participate in tumor initiation, progression, and metastasis (9). Therefore, dissecting the dysregulation of key genes associating with COAD may provide a new strategy for the discovery of potential treatment targets (10).

Chromobox protein homolog 3 (CBX3) is a member of the heterochromatin protein 1 (HP1) family and is involved in cell differentiation, growth, and transcriptional regulation (11). Previous studies have demonstrated that CBX3 is highly overexpressed in many cancer types and is associated with tumorigenesis and treatment resistance (12-14). Recently, several studies have demonstrated that CBX family is a potential prognostic biomarker for colon tumor $(15,16)$. For instance, Ma et al. (17) conducted an in vitro assay to reveal that $\mathrm{CBX} 3$ could regulate tumor cell proliferation. Knocking down CBX3 led to an increase in apoptosis and cell cycle arrest in osteosarcoma cells. A similar result was also observed by Lin et al. in gastric cancer (13), where knocking down CBX3 significantly inhibited the malignant phenotype and migration. Collectively, these findings suggested that CBX3 was associated with tumor progression. However, the function of CBX3 in COAD, especially its potential influence on tumor progression and metastasis, has not been verified. We designed this study to elucidate the association between CBX3 and metastasis in COAD. We present the following article in accordance with the REMARK reporting checklist (available at https://jgo.amegroups.com/article/ view/10.21037/jgo-22-97/rc).

\section{Methods}

\section{Data collection}

The Cancer Genome Atlas (TCGA) COAD datasets were available from University of California Santa Cruz (UCSC) Xena (https://xena.ucsc.edu) and included a messenger RNA (mRNA) expression profile (Illumina HiSeq 2000 RNA sequencing [RNA-seq]; 329 samples), Illumina 450K DNA methylation arrays, and a phenotype profile (545 samples). After intersecting the 2 profiles, 326 samples remained for inclusion in the analysis. The datasets GSE132465 (18), GSE77953 (19), GSE14297 (20), and GSE173858 were obtained from the Gene Expression Omnibus (GEO, https:// www.ncbi.nlm.nih.gov/geo). All samples were treatment free. The GSE132465 dataset consisted of 63,689 cells derived from 33 samples. Ten out of 33 samples (16,404 cells) from GSE132465 were labeled as normal mucosa. Therefore, these cells were filtered out of the analysis. Detailed information of samples and preparation methods are given in the GEO database. The study was conducted in accordance with the Declaration of Helsinki (as revised in 2013).

\section{Single-cell RNA-seq data analysis}

Single-cell RNA-seq (scRNA-seq) data analysis was performed by the Seurat package in $\mathrm{R}$ (version 3.2.3) (21). All cells were first subjected to clustering through the K-means algorithm with parameter resolution $=0.2$. Malignant-like cell clusters were then identified by tumor markers, including epithelial cell adhesion molecule (EpCAM) and keratin 8 (KRT8). Only the clusters whose proportion of cells expressing EpCAM and KRT8 was greater than 50\% were labeled as malignant-like tumor cell clusters.

\section{ChIP-seq data analysis}

Cbromatin immunoprecipitation sequencing (ChIP-seq) data were available from ChIP-Atlas (https://chip-atlas.org). Integrative Genomics Viewer (IGV, version 2.6.2) was used to perform data visualization. 
A

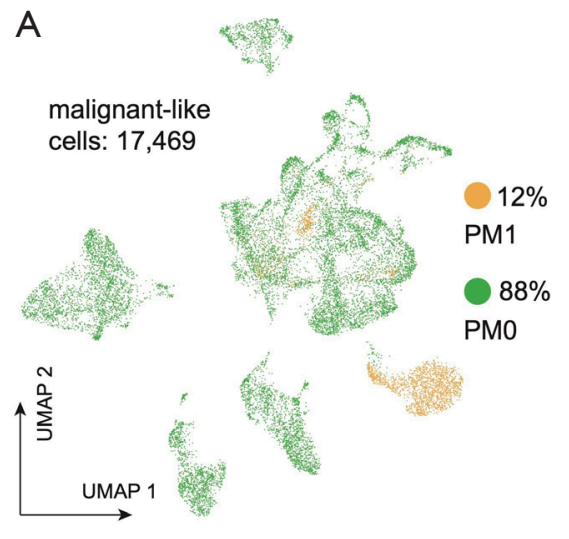

B

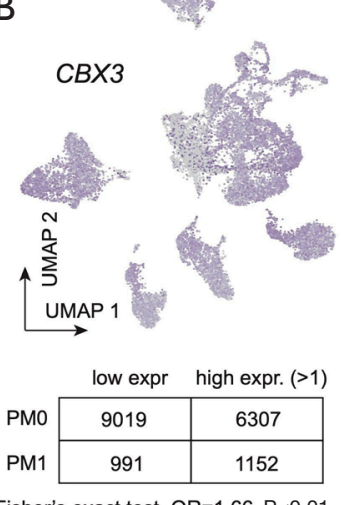

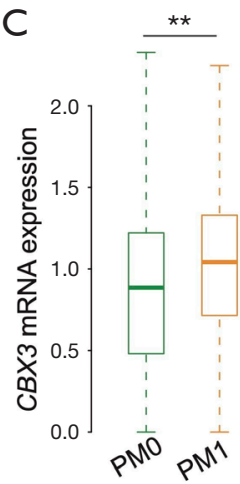

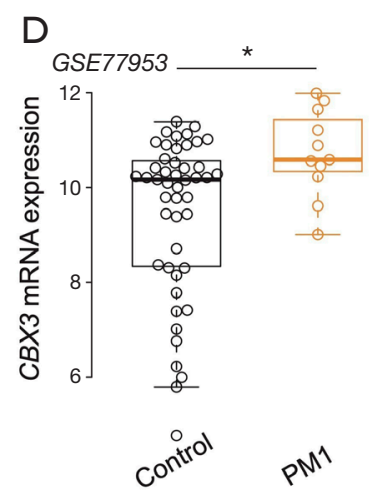

E

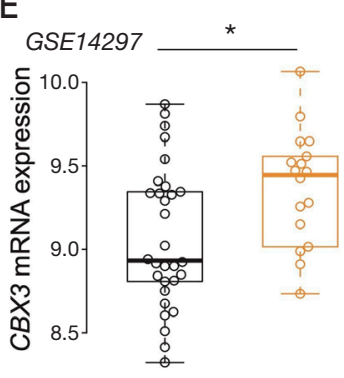

$\mathrm{F}$
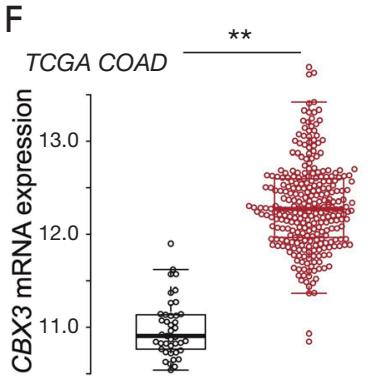

control $\quad \mathrm{N}^{\mathrm{N}}$

Control COAD

Wilcoxon's rank-sum test, $\mathrm{P}$ value $<0.05:^{*},<0.01:^{* \star}$.

Figure 1 Overexpression of CBX3 is associated with metastasis. (A) t-SNE plot of primary tumor (PM0, green dots) and metastatic tumor (PM1, orange dots) cells based on scRNA-seq dataset. (B) Top: Distribution of CBX3 mRNA level across malignant-like cells. Bottom: Number of cells in indicated groups. (C) Comparison of CBX3 mRNA levels in PM0 and PM1 groups. (D,E) Comparison of CBX3 mRNA levels in control and PM1 groups. (F) Comparison of CBX3 mRNA levels in control and COAD. CBX3, chromobox protein homolog 3; COAD, colon adenocarcinoma. Wilcoxon's rank-sum test, *, $\mathrm{P}<0.05$; **, $\mathrm{P}<0.01$.

\section{Statistical analysis}

We used the Wilcoxon rank-sum test and Student's $t$-test (for continuous quantitative variables) or Fisher's exact test (for categorical variables) to compare differences between groups. Survival analysis methods, including Kaplan-Meier plots and Cox proportional hazards models, were used to estimate the effect of CBX3 on clinical outcome. Analyses were carried out using statistical software R. All figures were plotted by ggplot2 package.

\section{Results}

\section{CBX3 is overexpressed in primary metastatic $C O A D$}

To explore the differential expression of CBX3 in primary COAD with metastasis (PM1) and without metastasis (PM0), we first collected an scRNA-seq profile consisting of
47,285 cells derived from 23 COAD samples (GSE132465), including 2 diagnosed with PM1. We clustered these cells using K-means algorithm and filtered out tumor cells through EpCAM and KRT8 (18), resulting in 17,469 malignant-like cells (Figure 1A). PM1 cells comprised $12 \%$ of these malignant-like cells. The proportion of PM1 cells with high expression of CBX3 was $53.8 \%$, which was significantly higher than that of PM0 cells $(41.2 \%)$ [Fisher's exact test, odds ratio $(\mathrm{OR})=1.66, \mathrm{P}<0.01]$ (Figure $1 B$ ). The average mRNA expression of CBX3 in PM1 cells was also significantly higher than that in PM0 cells (Student's $t$-test, $\mathrm{P}<0.01$ ) (Figure $1 C$ ). This finding was further confirmed by 2 independent data sets derived from Qu et al. (19) and Stange et al. (20), in which CBX3 mRNA level in PM1 was also higher than that of the control group (Student's $t$-test, $\mathrm{P}<0.05$ ) (Figure $1 D, 1 E$ ). Finally, we analyzed the expression of CBX3 in the mRNA profile from the TCGA COAD 
dataset and found that the CBX3 was significantly expressed in tumor tissues compared with adjacent normal tissues or peripheral blood $(\mathrm{P}<0.01)$ (Figure $1 F)$. Collectively, these findings suggested that $\mathrm{CBX} 3$ was associated with $\mathrm{COAD}$ metastasis.

\section{CBX3 is associated with regulating CCT6A, LSM5, and GGCT}

Next, we considered how CBX3 performed its role in COAD. A coexpression profile for $\mathrm{CBX} 3$ and genes based on the TCGA COAD dataset was generated through Pearson's correlation test in total online: https://cdn. amegroups.cn/static/public/jgo-22-97-1.pdf. We focused on analyzing the top 5 genes with the highest correlation coefficients, including chaperonin-containing TCP1 subunit 6A (CCT6A), Like Sm 5 (LSM5), gammaglutamylcyclotransferase (GGCT), nuclear factor (erythroid 2)-like factor 3 (NFE2L3), and DEAD-Box Helicase 56 (DDX56) (Figure 2A). Interestingly, CCT6A is a member of the chaperonin-containing TCP1 complex. In a study conducted by Ying et al. (22), CCT6A was found to act as an inhibitor to suppress SMAD family member 2 (SMAD2) and switch transforming growth factor beta (TGF- $\beta$ ) signaling to promote metastasis. GGCT is involved in glutathione homeostasis, and it has been reported (23) that knocking down GGCT could efficiently reduce colony formation and proliferation of tumor cells.

We further analyzed the 5 genes in the scRNAseq dataset and confirmed that all showed a significant correlation with $\mathrm{CBX} 3$ expression $(r>0.8, \mathrm{P}<0.01)$ across malignant-like cells (Figure 2B). Encouraged by these findings, we then considered whether CBX3 could regulate these genes. We performed ChIP-seq analysis based on a colon cancer cell line (SRX190214) derived from ChIPAtlas (24). Notably, with the exception of NFE2L3, the genes were detected with CBX3 binding sites upstream of the promoter $(<1 \mathrm{~KB})$ (Figure $2 C)$. The binding pattern predicted by Multiple EM for Motif Elicitation (MEME) (25) is shown in Figure 2D. We also collected an mRNA profile derived from a set of cell lines treated with CBX3 small interfering RNA (siRNA) or control siRNA (GSE173858). As expected, we found that the average of 5 genes showed a significant decrease in the siCBX3 group (Wilcoxon's rank-sum test, $\mathrm{P}<0.05$ ) (Figure $2 E$ ), suggesting that $\mathrm{CBX} 3$ could regulate the mRNA level of these genes. Further, by exploring the expression of these genes in 2 independent data sets derived from Qu et al. (19) and Stange et al. (20), we found that except for DDX56, the genes showed a higher mRNA level in the PM1 group (Figure 2F). Taken together, these findings implied that $\mathrm{CBX} 3$ regulated CCT6A, LSM5, and GGCT expression in COAD.

\section{CBX3 is involved in glutathione metabolism}

Since we had identified a series of genes potentially regulated by $\mathrm{CBX} 3$, we further characterized the function of these genes. Kyoto Encyclopedia of Genes and Genomes (KEGG) pathway enrichment analysis [via Enrichr (26)] demonstrated that glutathione metabolism, which is an important antioxidant involved in regulating carcinogenesis and progression (27), was significantly associated with the gene set $(\mathrm{P}=0.011)$ (Figure $3 A)$. Moreover, we constructed a gene-gene interaction network based on these genes and CBX3 through the GeneMANIA tool (28) (Figure 3B). The interaction network showed 2 distinct modules, including the TCP1 complex (CCT), and the LSM family (Figure $3 B$ ). CCT is involved in intracellular protein folding. Recent studies have revealed that CCT could regulate several signaling pathways to promote cancer metastasis, such as the $W n t 7 b / \beta$-catenin pathway and the protein kinase $\mathrm{B}(\mathrm{AKT}) / \mathrm{glycogen}$ synthase kinase 3 beta $($ GSK3 $\beta) / \beta$-catenin pathway $(29,30)$. The LSM family participates in splicing and cytoplasmic mRNA degradation. It has been reported that the LSM family could promote epithelial-mesenchymal-transition (EMT) and drive tumor metastasis (31). By integrating all of the genes on the network into KEGG pathway enrichment analysis, we found that glutathione metabolism was presented with high significance $(\mathrm{P}=6.04 \mathrm{e}-7)$ (Figure $3 \mathrm{C})$, suggesting that CBX3 may drive glutathione metabolism. To validate the impact of CBX3 on glutathione metabolism, we applied the analysis to the mRNA profile derived from a set of cell lines treated with CBX3 small interfering RNA (siRNA) or control siRNA (GSE173858). As expected, we found that the glutathione metabolism signature score, which was predicted by single-sample Gene Set Enrichment Analysis (ssGSEA) algorithm (32), showed a remarkable decrease in the CBX3 siRNA group (Figure 3D).

\section{CBX3 is involved in glycosphingolipid metabolism}

It is well known that HP1 is involved in controlling DNA methylation and regulating the impact of DNA methylation on alternative splicing. Hence, we explored the specific DNA methylations associated with CBX3. We divided 

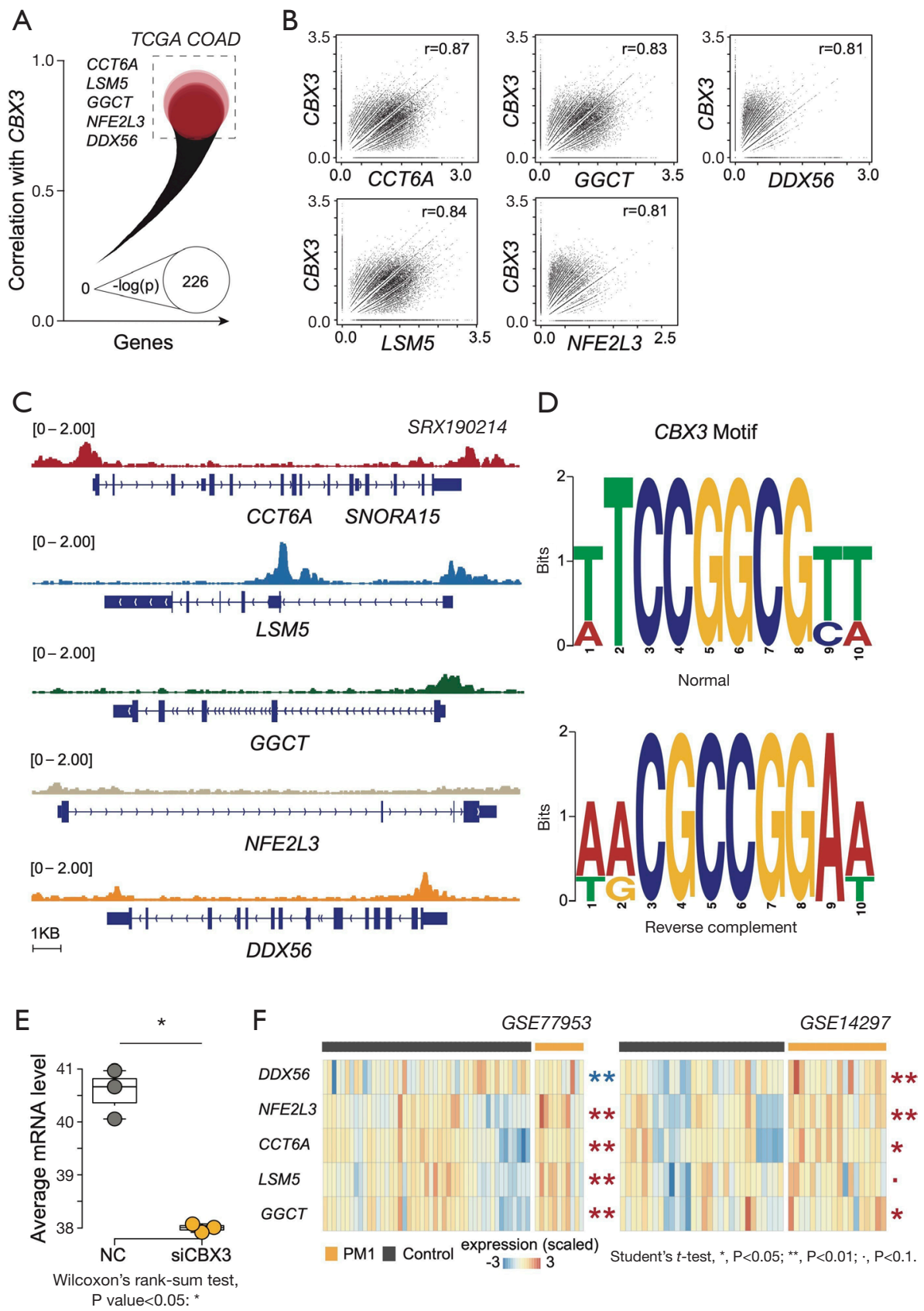

Figure 2 Analysis of downstream regulation of CBX3. (A) Correlation between CBX3 and other gene mRNA levels across TCGA COAD dataset. The $\mathrm{Y}$ axis represents the correlation coefficient. The size of data point represents the significance of correlation. Top 5 genes with the highest significance are in red. (B) Correlation between CBX3 and the indicated genes across scRNA-seq dataset. (C) ChIP-Seq analysis of CBX3 in colon cell line. The representative Integrative Genomics Viewer (IGV) tracks at the indicated gene locus show the distribution of peaks upstream of the transcription start site (TSS) (<5 KB). (D) Binding motif of CBX3 predicted by MEME. (E) Comparison of average mRNA level of five genes between NC and siCBX3 group. Wilcoxon's rank-sum test, * $\mathrm{P}<0.05$. (F) Comparison of indicated genes mRNA level between PM1 and control group. Student's $t$-test, ${ }^{*}, \mathrm{P}<0.05 ;{ }^{* *}, \mathrm{P}<0.01 ; \cdot, \mathrm{P}<0.1$. CBX3, chromobox protein homolog 3 ; COAD, colon adenocarcinoma. 

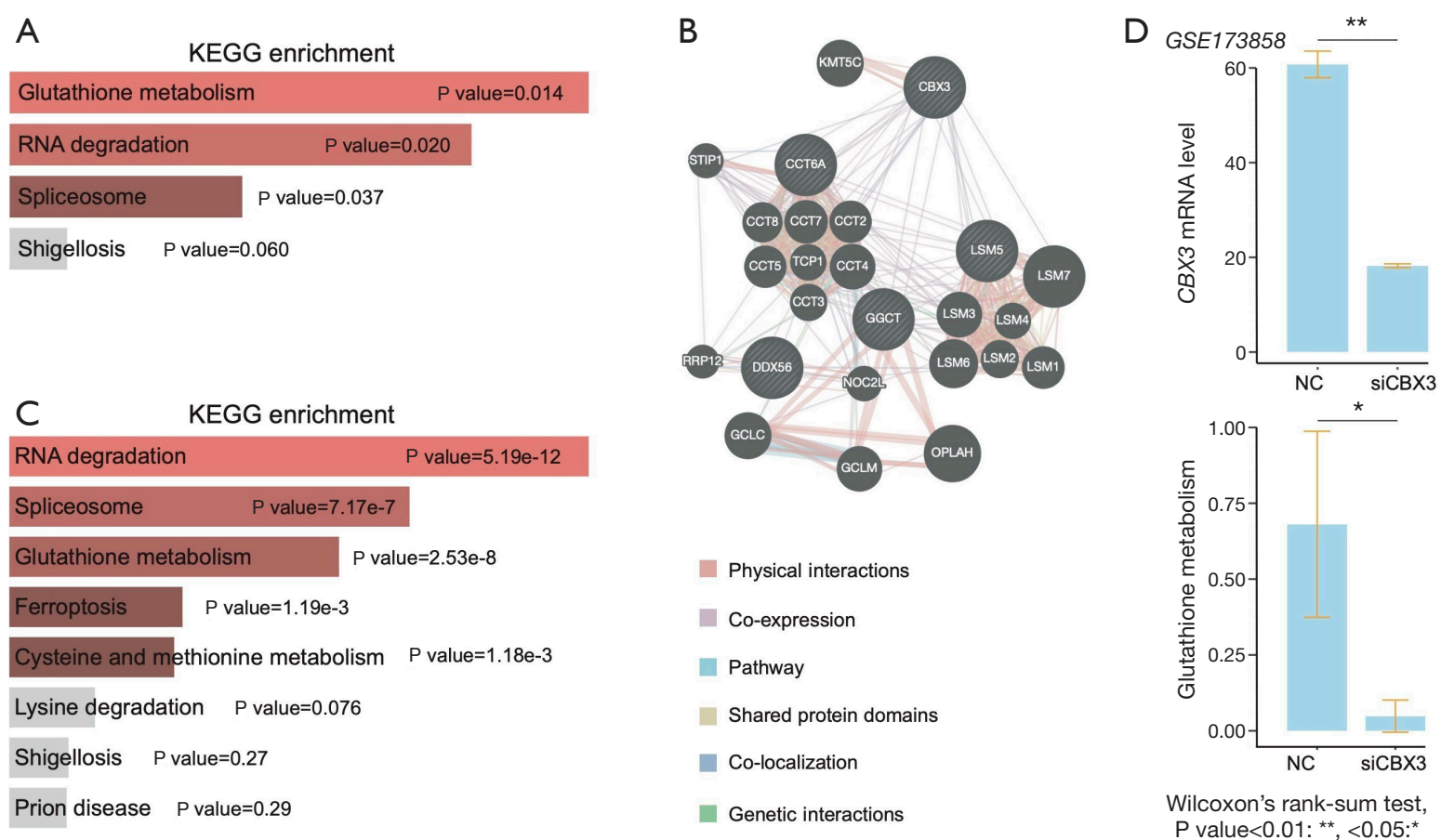

Figure 3 CBX3 is associated with glutathione metabolism. (A) KEGG enrichment analysis via Enrichr. (B) Interaction network analysis via GeneMANIA. (C) KEGG enrichment analysis based on gene set in interaction network via Enrichr. (D) Top: Comparison of CBX3 mRNA levels among the indicated groups. Bottom: Comparison of glutathione metabolism enrichment scores among the indicated groups. Wilcoxon's rank-sum test, ${ }^{*}, \mathrm{P}<0.05 ;{ }^{* *}, \mathrm{P}<0.01$. NC, negative control; CBX3, chromobox protein homolog 3.

the samples from TCGA COAD into 2 groups according to the mRNA level of CBX3. By performing Wilcoxon's rank-sum test analysis, we identified 12 hypermethylation probes relating to 10 genes (Table 1). Interestingly, these genes were significantly enriched on glycosphingolipid biosynthesis ( $\mathrm{P}=0.0223$ ) (Figure $4 A)$. Glycosphingolipids play an important role as receptors in cell invasion. Several studies have demonstrated that cells conduct epithelialto-mesenchymal transition by dynamically decreasing glycosphingolipid biosynthesis. Furthermore, analysis based on TCGA COAD showed that CBX3 mRNA level was negatively correlated with glycosphingolipid metabolism $(r=-0.58, \mathrm{P}<0.01)$ (Figure $4 B)$, suggesting that $\mathrm{CBX} 3$ may be associated with the suppression of glycosphingolipid metabolism.

\section{Patients with high CBX3 expression show poor prognosis}

Finally, we evaluated the clinical features of CBX3. By dividing cases in the TCGA COAD dataset into 2 groups based on CBX3 level (high group: top 33\%; low group: bottom $67 \%$ ), we found that cases with high CBX3 levels had a nearly 2 -fold shorter median survival time than those in the control group [hazard ratio $(\mathrm{HR})=1.59$; likelihood ratio test, $\mathrm{P}=0.04$ ] (Figure $5 \mathrm{~A}$ ). Additionally, when we focused solely on disease-free interval time, we found that the cases with high CBX3 still had a shorter time than the control group $(\mathrm{HR}=2.20$, likelihood ratio test, $\mathrm{P}=0.1)$ (Figure $5 B$ ). Further, multivariate analysis controlling for age, gender, and histological type suggested that CBX3 was an independent prognostic factor $[\mathrm{HR}=1.64,95 \%$ confidence interval (CI): 1.03-2.6, $\mathrm{P}=0.036$ ] (Figure 5C).

\section{Discussion}

Metastasis is the primary cause of COAD mortality (33), and the identification of potential targets for inhibiting progression is urgently needed. The COAD metastasis may be caused by a series of processes, including angiogenesis, microenvironment remodeling, the epithelial-mesenchymal transition, etc. (1). Especially, the reshaping of the tumor microenvironment is considered to be an essential component during tumor metastasis (34), which may be regulated by TGF- $\beta$ and other cytokines $(35,36)$. On the 
Table 1 High CBX3 associated DNA methylation probes

\begin{tabular}{|c|c|c|c|c|c|}
\hline Chromosome & Position & Strand & Name & Relation to island & Gene name \\
\hline 3 & 134083080 & + & cg05304177 & Island & AMOTL2 \\
\hline 11 & 64108322 & - & cg12043314 & N_Shore & $C C D C 88 B$ \\
\hline 2 & 27165781 & + & cg14455516 & OpenSea & DPYSL5 \\
\hline 1 & 154971922 & + & $\operatorname{cg} 10445911$ & Island & - \\
\hline 6 & 33244976 & - & cg21333861 & Island & B3GALT4 \\
\hline 19 & 1248287 & + & cg02986494 & Island & MIDN \\
\hline 19 & 18118079 & + & cg18096253 & Island & $A R R D C 2$ \\
\hline 16 & 88803885 & - & cg00973732 & Island & FAM38A \\
\hline
\end{tabular}

CBX3, chromobox protein homolog 3 .
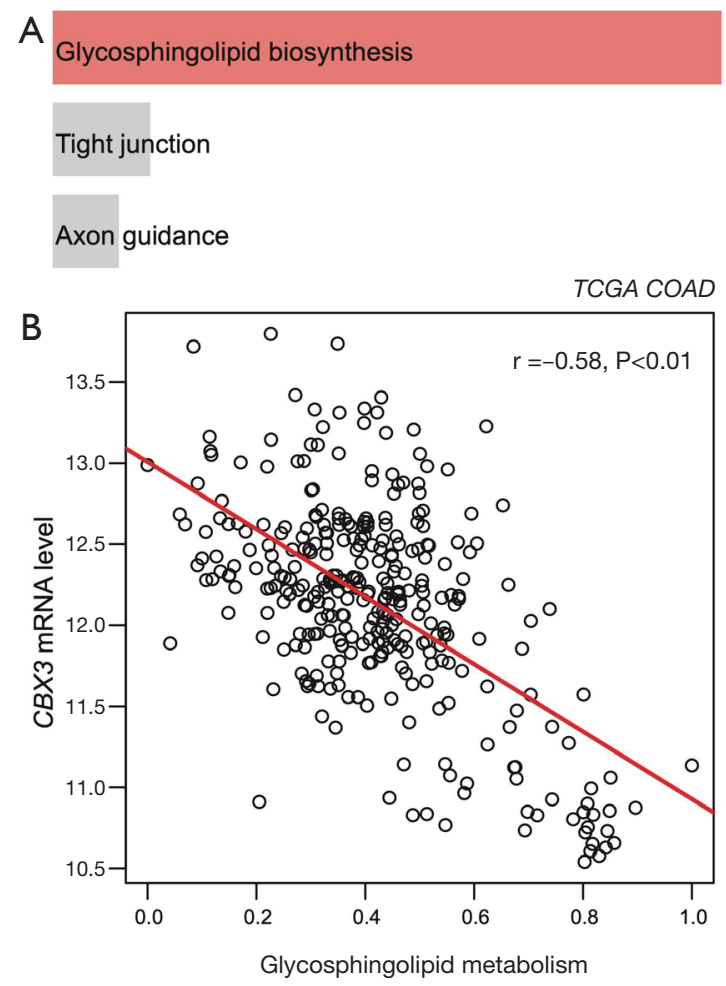

Figure 4 CBX3 is associated with glycosphingolipid metabolism. (A) KEGG enrichment analysis via Enrichr. (B) Correlation between CBX3 mRNA level and glycosphingolipid metabolism enrichment score. CBX3, chromobox protein homolog 3 . one hand, these cytokines function as an immunosuppressive factor to inhibit the activity of immune cells, such as natural killer cells, etc. $(1,36)$. On the other hand, these molecules also accelerate epithelial-mesenchymal transition to promote tumor metastasis (37). Hence, it is essential to identify the potential upstream regulators, which may help deepen our understanding of such a metastasis process.

Our study suggested that CBX3 was associated with COAD metastasis. Patients with high CBX3 had a shorter overall survival time. However, the downstream regulation axis of $\mathrm{CBX} 3$-driven metastasis remains to be further explained. One possible clue promoting metastasis may be attributed to CCT6A since it is a component of the chaperonin containing TCP1 complex and has been confirmed to switch TGF- $\beta$ signaling to promote metastasis. In our study, the results of ChIP-seq analysis coupled with correlation analysis indicated that $\mathrm{CBX} 3$ could regulate CCT6A expression, suggesting that the CBX3CCT6A regulation pathway may be a potential target for inhibiting tumor metastasis.

Increasingly, studies are being drawn to the impact of tumor metabolism on metastasis (38). Our study suggested that glutathione metabolism was associated with a CBX3driven interaction network. Glutathione is a key antioxidant associated with regulating carcinogenesis and progression. It has been reported that tumor cells require certain 

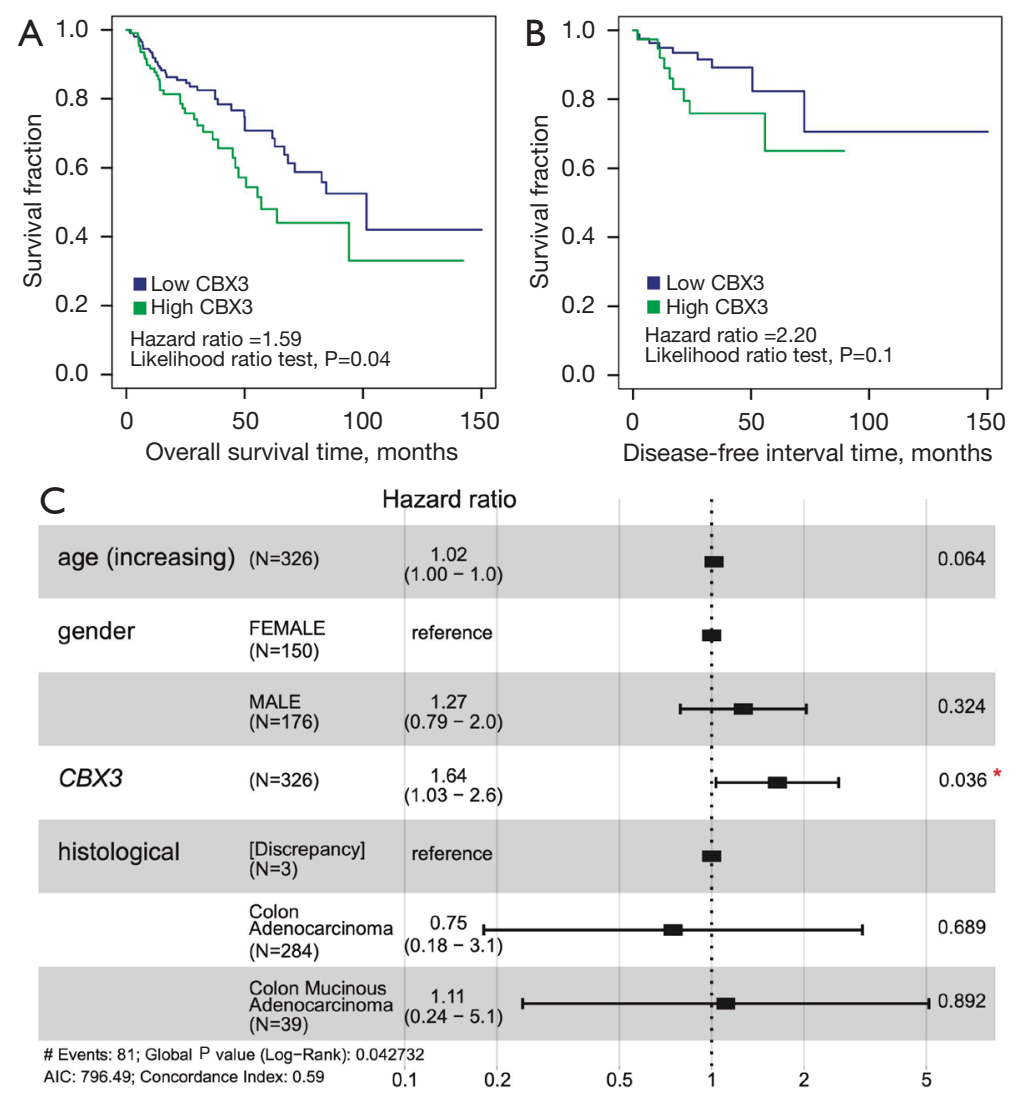

Figure 5 CBX3 is associated with poor prognosis. Overall survival (A) and disease-free interval (B) time analysis of COAD cases for high and low CBX3 groups. (C) Multivariable Cox regression analysis of overall survival of TCGA COAD cases on 4 prominent variables, as indicated. CBX3, chromobox protein homolog 3; COAD, colon adenocarcinoma. Wilcoxon's rank-sum test, ${ }^{*}, \mathrm{P}<0.05$.

metabolic products, such as glutathione, to maintain reactive oxygen species (ROS) levels for supporting survival in circulation (38). Moreover, glycosphingolipid metabolism was negatively correlated with CBX3 expression, which may be associated with CBX3's role in controlling DNA methylation. Many studies have shown that glycosphingolipid metabolism has low activity during the epithelial-to-mesenchymal transition process.

Since the aim of this study was using bioinformatics analysis to integrate multi-omics data and systematically explore the putative function of $\mathrm{CBX} 3$ on promoting COAD progression. One limitation of our study is a lack of in vivo and in vitro experiments to validate the findings above. In the future, we will conduct more experiments to further identify and validate the key molecules found in our study which may participate in COAD metastasis.

In conclusion, our work showed that CBX3 overexpression was associated with COAD metastasis. The CBX3 downstream regulation network involved TCP1 complex, the LSM family, and glutathione metabolism, which may provide a potential target for suppressing tumor metastasis.

\section{Acknowledgments}

All the authors appreciate Professor Qianghu Wang's group, the Department of Bioinformatics, Nanjing Medical University, for the valuable advice on bioinformatics and statistical analysis.

Funding: None.

\section{Footnote}

Reporting Checklist: The authors have completed the REMARK reporting checklist. Available at https://jgo. amegroups.com/article/view/10.21037/jgo-22-97/rc

Conflicts of Interest: All authors have completed the ICMJE 
uniform disclosure form (available at https://jgo.amegroups. com/article/view/10.21037/jgo-22-97/coif). The authors have no conflicts of interest to declare.

Ethical Statement: The authors are accountable for all aspects of the work in ensuring that questions related to the accuracy or integrity of any part of the work are appropriately investigated and resolved. The study was conducted in accordance with the Declaration of Helsinki (as revised in 2013).

Open Access Statement: This is an Open Access article distributed in accordance with the Creative Commons Attribution-NonCommercial-NoDerivs 4.0 International License (CC BY-NC-ND 4.0), which permits the noncommercial replication and distribution of the article with the strict proviso that no changes or edits are made and the original work is properly cited (including links to both the formal publication through the relevant DOI and the license). See: https://creativecommons.org/licenses/by-nc-nd/4.0/.

\section{References}

1. Pretzsch E, Bösch F, Neumann J, et al. Mechanisms of Metastasis in Colorectal Cancer and Metastatic Organotropism: Hematogenous versus Peritoneal Spread. J Oncol 2019;2019:7407190.

2. Vera R, González-Flores E, Rubio C, et al. Multidisciplinary management of liver metastases in patients with colorectal cancer: a consensus of SEOM, AEC, SEOR, SERVEI, and SEMNIM. Clin Transl Oncol 2020;22:647-62.

3. Powell SM, Zilz N, Beazer-Barclay Y, et al. APC mutations occur early during colorectal tumorigenesis. Nature 1992;359:235-7.

4. Iacopetta B. TP53 mutation in colorectal cancer. Hum Mutat 2003;21:271-6.

5. Lièvre $\mathrm{A}$, Bachet JB, Le Corre D, et al. KRAS mutation status is predictive of response to cetuximab therapy in colorectal cancer. Cancer Res 2006;66:3992-5.

6. Rokavec M, Horst D, Hermeking H. Cellular Model of Colon Cancer Progression Reveals Signatures of mRNAs, miRNA, lncRNAs, and Epigenetic Modifications Associated with Metastasis. Cancer Res 2017;77:1854-67.

7. Aiello NM, Stanger BZ. Echoes of the embryo: using the developmental biology toolkit to study cancer. Dis Model Mech 2016;9:105-14.

8. Manzo G. Similarities Between Embryo Development and
Cancer Process Suggest New Strategies for Research and Therapy of Tumors: A New Point of View. Front Cell Dev Biol 2019;7:20.

9. Zhan T, Rindtorff N, Boutros $M$. Wnt signaling in cancer. Oncogene 2017;36:1461-73.

10. Davies H, Bignell GR, Cox C, et al. Mutations of the BRAF gene in human cancer. Nature 2002;417:949-54.

11. Huang C, Su T, Xue Y, et al. Cbx3 maintains lineage specificity during neural differentiation. Genes Dev 2017;31:241-6.

12. Chen LY, Cheng CS, Qu C, et al. CBX3 promotes proliferation and regulates glycolysis via suppressing FBP1 in pancreatic cancer. Biochem Biophys Res Commun 2018;500:691-7.

13. Lin H, Lian J, Xia L, et al. CBX3 Promotes Gastric Cancer Progression and Affects Factors Related to Immunotherapeutic Responses. Cancer Manag Res 2020;12:10113-25.

14. Chang SC, Lai YC, Chen YC, et al. CBX3/ heterochromatin protein 1 gamma is significantly upregulated in patients with non-small cell lung cancer. Asia Pac J Clin Oncol 2018;14:e283-8.

15. Zhou H, Xiong Y, Liu Z, et al. Expression and prognostic significance of $\mathrm{CBX} 2$ in colorectal cancer: database mining for CBX family members in malignancies and vitro analyses. Cancer Cell Int 2021;21:402.

16. Li Q, Pan Y, Cao Z, et al. Comprehensive Analysis of Prognostic Value and Immune Infiltration of Chromobox Family Members in Colorectal Cancer. Front Oncol 2020;10:582667.

17. Ma C, Nie XG, Wang YL, et al. CBX3 predicts an unfavorable prognosis and promotes tumorigenesis in osteosarcoma. Mol Med Rep 2019;19:4205-12.

18. Lee HO, Hong Y, Etlioglu HE, et al. Lineage-dependent gene expression programs influence the immune landscape of colorectal cancer. Nat Genet 2020;52:594-603.

19. Qu X, Sandmann T, Frierson H Jr, et al. Integrated genomic analysis of colorectal cancer progression reveals activation of EGFR through demethylation of the EREG promoter. Oncogene 2016;35:6403-15.

20. Stange DE, Engel F, Longerich T, et al. Expression of an ASCL2 related stem cell signature and IGF2 in colorectal cancer liver metastases with $11 \mathrm{p} 15.5$ gain. Gut 2010;59:1236-44.

21. Stuart T, Butler A, Hoffman P, et al. Comprehensive Integration of Single-Cell Data. Cell 2019;177:18881902.e21.

22. Ying Z, Tian H, Li Y, et al. CCT6A suppresses SMAD2 
and promotes prometastatic TGF-beta signaling. J Clin Invest 2017;127:1725-40.

23. Zhang W, Chen L, Xiang H, et al. Knockdown of GGCT inhibits cell proliferation and induces late apoptosis in human gastric cancer. BMC Biochem 2016;17:19.

24. Oki S, Ohta T, Shioi G, et al. ChIP-Atlas: a data-mining suite powered by full integration of public ChIP-seq data. EMBO Rep 2018;19:e46255.

25. Bailey TL, Johnson J, Grant CE, et al. The MEME Suite. Nucleic Acids Res 2015;43:W39-49.

26. Xie Z, Bailey A, Kuleshov MV, et al. Gene Set Knowledge Discovery with Enrichr. Curr Protoc 2021;1:e90.

27. Narayanankutty A, Job JT, Narayanankutty V. Glutathione, an Antioxidant Tripeptide: Dual Roles in Carcinogenesis and Chemoprevention. Curr Protein Pept Sci 2019;20:907-17.

28. Warde-Farley D, Donaldson SL, Comes O, et al. The GeneMANIA prediction server: biological network integration for gene prioritization and predicting gene function. Nucleic Acids Res 2010;38:W214-20.

29. Chang YX, Lin YF, Chen CL, et al. ChaperoninContaining TCP-1 Promotes Cancer Chemoresistance and Metastasis through the AKT-GSK3beta-betaCatenin and XIAP-Survivin Pathways. Cancers (Basel) 2020;12:3865.

30. Tang N, Cai X, Peng L, et al. TCP1 regulates Wnt7b/ beta-catenin pathway through P53 to influence the

Cite this article as: Zhong X, Ni J, Jia Z, Yan H, Zhang Y, Liu Y. $C B X 3$ is associated with metastasis and glutathione/ glycosphingolipid metabolism in colon adenocarcinoma. J Gastrointest Oncol 2022;13(1):246-255. doi: 10.21037/jgo-22-97 proliferation and migration of hepatocellular carcinoma cells. Signal Transduct Target Ther 2020;5:169.

31. Little EC, Camp ER, Wang C, et al. The CaSm (LSm1) oncogene promotes transformation, chemoresistance and metastasis of pancreatic cancer cells. Oncogenesis 2016;5:e182.

32. Barbie DA, Tamayo P, Boehm JS, et al. Systematic RNA interference reveals that oncogenic KRAS-driven cancers require TBK1. Nature 2009;462:108-12.

33. Vatandoust S, Price TJ, Karapetis CS. Colorectal cancer: Metastases to a single organ. World J Gastroenterol 2015;21:11767-76.

34. Mariani F, Sena P, Roncucci L. Inflammatory pathways in the early steps of colorectal cancer development. World J Gastroenterol 2014;20:9716-31.

35. Lampropoulos P, Zizi-Sermpetzoglou A, Rizos S, et al. TGF-beta signalling in colon carcinogenesis. Cancer Lett 2012;314:1-7.

36. West NR, McCuaig S, Franchini F, et al. Emerging cytokine networks in colorectal cancer. Nat Rev Immunol 2015;15:615-29.

37. Xiao J, Zhou N, Li Y, et al. PEITC inhibits the invasion and migration of colorectal cancer cells by blocking TGF-beta-induced EMT. Biomed Pharmacother 2020;130:110743.

38. Bergers G, Fendt SM. The metabolism of cancer cells during metastasis. Nat Rev Cancer 2021;21:162-80. 